\title{
Optimal Control for One-Qubit Quantum Sensing
}

\author{
F. Poggiali, ${ }^{1,2}$ P. Cappellaro, ${ }^{1,3}$ and N. Fabbri ${ }^{1,2, *}$ \\ ${ }^{1}$ LENS European Laboratory for Non-Linear Spectroscopy, \\ Università di Firenze, I-50019 Sesto Fiorentino, Italy \\ ${ }^{2}$ INO-CNR Istituto Nazionale di Ottica del CNR, I-50019 Sesto Fiorentino, Italy \\ ${ }^{3}$ Department of Nuclear Science and Engineering, Massachusetts Institute of Technology, \\ Cambridge, Massachusetts 02139, USA
}

(Received 22 December 2017; revised manuscript received 10 April 2018; published 7 June 2018)

\begin{abstract}
Quantum systems can be exquisite sensors thanks to their sensitivity to external perturbations. This same characteristic also makes them fragile to external noise. Quantum control can tackle the challenge of protecting a quantum sensor from environmental noise, while strongly coupling the sensor with the field to be measured. As the compromise between these two conflicting requirements does not always have an intuitive solution, optimal control based on a numerical search could prove very effective. Here, we adapt optimal control theory to the quantum-sensing scenario by introducing a cost function that, unlike the usual fidelity of operation, correctly takes into account both the field to be measured and the environmental noise. We experimentally implement this novel control paradigm using a nitrogen vacancy center in diamond, finding improved sensitivity to a broad set of time-varying fields. The demonstrated robustness and efficiency of the numerical optimization, as well as the sensitivity advantage it bestows, will prove beneficial to many quantum-sensing applications.
\end{abstract}

DOI: 10.1103/PhysRevX.8.021059

Subject Areas: Quantum Physics

\section{INTRODUCTION}

Quantum control has been demonstrated to be a crucial tool both in quantum information processing [1] and in quantum sensing $[2,3]$ on a variety of experimental platforms, ranging from trapped ions $[4,5]$ to ultracold atoms $[6,7]$ and superconducting qubits $[8,9]$, as well as nuclear $[10,11]$ and electronic spin qubits [12,13]. Quantum sensing poses peculiar challenges to control, as sensor qubits need to interact strongly with the target field to be probed, but this also leads to undesired coupling with external noise of the same nature of the target field, which often gives rise to either energy losses or decoherence. A paradigmatic scenario is when one wants to measure a frequency shift of a spin qubit sensor, as due to a magnetic field, in the presence of magnetic dephasing noise.

Optimal control theory $[14,15]$ exploits numerical optimization methods [16-20] to find the best control fields that steer the dynamics of a system towards the desired goal. Quantum optimal control has been successfully applied in the case of one- and few-body systems [21-27], as well as in

\footnotetext{
*fabbri@lens.unifi.it
}

Published by the American Physical Society under the terms of the Creative Commons Attribution 4.0 International license. Further distribution of this work must maintain attribution to the author(s) and the published article's title, journal citation, and DOI. ensembles [28] and correlated many-body quantum systems $[7,29,30]$.

Typically, the optimal control problem involves the search for the optimal transformation that, given a system Hamiltonian $\mathcal{H}$ dependent on a set of time-dependent control fields, drives the system from an initial state into a target state, whose desired properties are expressed by a cost function $\mathcal{F}$ that one wants to minimize. Often this means maximizing the fidelity of the unitary operation, which describes this transformation, with the desired one.

However, the goal of quantum sensing is different. Since there is at most only some partial knowledge of the external field to be measured, the expected unitary dynamics is unknown, and thus the fidelity cannot be used to optimize control. In addition, quantum sensing is usually concerned with optimizing sensitivity, a quantity that intrinsically includes noise, also arising from the external environment.

Here, we devise and experimentally demonstrate a robust and efficient scheme for optimal control of a sensing qubit, which enhances its sensitivity to the amplitude of timevarying target fields. For this purpose, we use an unconventional optimization metric, the sensitivity, and develop a practical way of computing it (which allows for fast numerical searches). Furthermore, our search method includes, in the cost metric itself, the presence of an environment and the consequent decoherence induced on the qubit. While optimal control has been used before for sensing [31-33], the optimization was only targeted at 
improving the control fidelity and bandwidth, not the sensitivity itself.

Here, we tackle the complex task of measuring multichromatic ac target fields and different significant waveforms, such as trains of magnetic impulses, which are relevant for applications in biology, physiology, and neuroscience [34-37]. We show that, in these cases, optimal control demonstrates better performance than traditional dynamical decoupling since it allows for both a larger accumulation of the spin phase that encodes the field information and for an improved compensation of environment-induced decoherence, thus boosting the qubit's sensitivity and enabling detection of very weak magnetic fields.

\section{OPTIMAL CONTROL OF A QUBIT SENSOR WITH DEPHASING NOISE}

We use the electron spin states of a nitrogen vacancy (NV) center in diamond as a sensing qubit of time-varying magnetic fields in the presence of magnetic noise, which induces dephasing of the sensing qubit. While the NV center forms a spin $S=1$, a static bias magnetic field removes the degeneracy of levels with spin projection $S_{z}= \pm 1$, and a microwave (mw) excitation selectively addresses the $S_{z}=0 \rightarrow-1$ transition; therefore, the NV center can be effectively described as a single two-level system [38]. The electron spin qubit can be prepared in a well-defined initial state, coherently manipulated, and read out [40].

The spin qubit couples both with a time-dependent external field to be measured, and with environmental magnetic noise. We model those couplings with the Hamiltonian

$$
\mathcal{H}=\gamma b(t) \sigma_{z}+\gamma \beta(t) \sigma_{z},
$$

where the field to be measured has the general form $b(t)=b f(t)$, and $\beta(t)$ is a stochastic variable with power spectral density $S(\omega)$ in the frequency domain. $\gamma=2.80 \times$ $10^{4} \mathrm{~Hz} / \mu \mathrm{T}$ is the gyromagnetic ratio, and $\sigma_{z}$ is the $z$ component of the spin operator, $\hat{z}$ being the NV symmetry axis. Performing metrology means reaching a compromise between two conflicting tasks, i.e., minimizing the noise effects while maximizing the signal stemming from the field, during the sensing time. Here, in particular, we assume to know the temporal dependence $f(t)$ of the field, and we aim at measuring its amplitude $b$ (we are thus interested in a parameter estimation task). We note that by embedding our scheme in an adaptive strategy, one could also tackle waveforms of unknown time dependency [41].

While different control strategies can be used for sensing, here we consider control via pulsed dynamical decoupling, which is realized with a series of $\pi$ pulses that repeatedly flip the spin, thus reversing its evolution [42]. The control field can thus be described by a modulation function $y_{n}(t)$, with a sign switch at the position of each $\pi$ pulse, indicating the direction of time evolution, forward or backward. The squared Fourier transform of $y_{n}(t)$ defines the filter control function $Y_{n, T}(\omega)$.

The phase accumulated by the spin during the sensing time $T$, under the action of the control field, is

$$
\varphi_{n}(T)=\int_{0}^{T} \gamma b(t) y_{n}(t) d t \equiv b \phi_{n} .
$$

To read out the phase due to the target field, we embed the control sequence within a Ramsey interferometer, which enables the mapping of the phase accumulated into the observable population of the spin projection $S_{z}=-1$.

As said, during the sensing process, the sensor qubit is also subject to noise. In the case of the NV center, this is mainly due to the nuclear spin bath that generates a stochastic time-varying field. Therefore, the qubit acquires a random phase during its coherent evolution, which leads to a reduction of the observed population.

The state of the qubit after the sensing process is described, as in a Ramsey interferometer, by population and coherence of the density matrix

$$
\rho_{1,1}(T)=\frac{1}{2}, \quad \rho_{1,2}(T, b)=\frac{1}{2} e^{-i \varphi_{n}(T)} e^{-\chi_{n}(T)},
$$

where $\chi_{n}(T)$ is temporal coherence function, describing noise-induced decoherence, that also depends on the control field through $Y_{n, T}(\omega)$,

$$
\chi_{n}(T)=\int d \omega S(\omega)\left|Y_{n, T}(\omega)\right|^{2} /\left(\pi \omega^{2}\right) .
$$

Thus, a projective measurement on the $\sigma_{x}$ basis, $| \pm\rangle=(|0\rangle \pm|1\rangle) / \sqrt{2}$, gives a signal

$s(T)=\langle+|\rho(T, b)|+\rangle=\frac{1}{2}\left(1+e^{-\chi_{n}(T)} \cos \varphi_{n}(T)\right)$.

We point out that we treat the environment as a source of pure decoherence noise and further assume a classical bath described by its spectrum $S(\omega)$, as this allows the simple expression in Eq. (4) to describe its effects $[44,45]$ (see Appendix A 2 for further discussion of this approximation validity). While a classical bath is a good model for many physical systems [46-55], the same formalism can tackle more complex and quantum baths [56,57]. More generally, using Eq. (4) to calculate the environmental effects on the sensor only simplifies the numerical optimization, but a more complex noise model could also be taken into account.

To assess the quality of parameter estimation, as achievable under a given control protocol and within the experimental constraints, we can evaluate the Fisher information $F_{N}[43,58]$ associated with the measurement, 


$$
F_{N}=\sum_{x} \frac{1}{p_{N}(x \mid b)}\left(\frac{\partial p_{N}(x \mid b)}{\partial b}\right)^{2}
$$

Here, $p_{N}(x \mid b)=\operatorname{Tr}\left[E_{x}^{(N)} \rho_{b}^{\otimes^{N}}\right]$ are conditional probabilities of obtaining $x$ as a measurement result for a given field $b$ over $N$ repeated measurements, $E_{x}$ being the measurement estimator and $\rho$ the density matrix of each independent copy of the system. Sensitivity, that is, the minimum detectable signal per unit time, is simply related to the Fisher information by

$$
\eta=\frac{\sqrt{\mathbb{T}}}{\sqrt{N F_{N}}},
$$

where $\mathbb{T}=N T$ is the total sensing experiment time. For the one-qubit sensing schemes we are considering, this reduces to

$$
\eta=\min \left\{\frac{\Delta s}{\partial_{b} s}\right\} \sqrt{T}=\frac{e^{\chi_{n}(T)}}{\left|\phi_{n}\right|} \sqrt{T} .
$$

This is indeed the cost function that we want to minimize. In practice, for a given field $b(t)$, we are searching for the optimal control field that steers the spin trajectory of the electronic spin on the Bloch sphere in such a way that, while the accumulated phase $\varphi_{n}(T)$ is maximized, the effect of non-Markovian noise described by $\chi_{n}(T)$ is minimized.

For this purpose, we have designed a direct and fast search method that looks for the optimal modulation function $y_{n}^{\mathrm{opt}}(t)$ that minimizes the cost function $\eta$. We have investigated various multidimensional parameter spaces, up to dimension $M=51$, and analyzed which optimization parameters (e.g., total sensing time, $\pi$-pulse positions, signal phase, signal trigger time) provide the largest improvement without requiring excessive computational resources, as we detail in the following. The constraints of the parameter space are chosen to describe realistic experimental conditions. The search of the optimal control field is performed by means of a simplex (Nelder Mead) minimization numerical algorithm that allows us to reach global convergence in the parameter space, as illustrated in Fig. 1(b). The method requires a precise knowledge of the temporal coherence function of the electronic spin sensor, which depends on the noise spectrum induced by its spin bath, as detailed in the Appendix A 2.
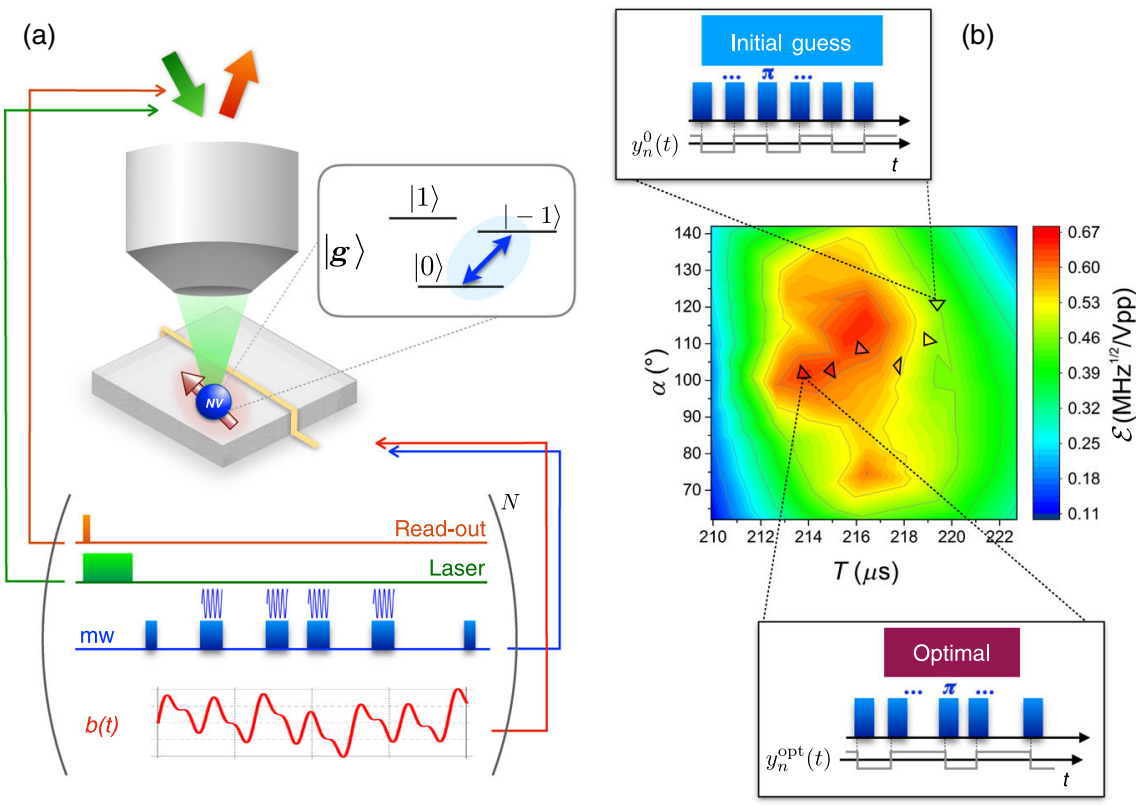

FIG. 1. One-qubit optimization strategy. (a) The electronic spin of a single NV center is optically initialized in the $|0\rangle$ state and read out after the sensing period by means of a confocal microscope. An antenna delivers both the resonant control field (mw) and the target magnetic field $b(t)$ to be measured, in the proximity of the spin qubit. Each measurement shot is repeated $N=2 \times 10^{5}$ times (see also Appendix A 1). (b) Illustration of the optimization protocol. The starting point is an initial guess for the control sequence described by a modulation function $y_{n}^{0}(t)$, which depends on a given number of parameters. While in the paper we consider more general cases, for the sake of simplicity, the central panel shows a search in a two-parameter space (sensing time $T$ and phase shift $\alpha$ ) for a Carr-Purcell (CP) control sequence used to detect a monochromatic ac field $b(t)=b \cos \left(2 \pi \nu_{0} t+\alpha\right)$, with frequency $\nu_{0}=20.5 \mathrm{kHz}$ and unknown amplitude $b$ to be measured. The map represents the experimentally measured inverse sensitivity $\mathcal{E}=C / \eta$ (see text). The algorithm computes the sensitivity $\eta$ under the initial control sequence and then produces and evaluates a number of other trial points $y_{n}^{(i)}(t)$ and moves in the multidimensional parameter space until global convergence is reached. The final point, described by $y_{n}^{\text {opt }}$, represents the optimal control sequence. 


\section{RESULTS}

The experiment is sketched in Fig. 1(a) (see also Appendix A 1). The electronic spin of a single NV center is optically initialized and read out by means of a confocal microscope. The spin qubit is coherently controlled with a resonant field and radiated with the off-resonance, timevarying, target magnetic field to be measured. We obtain the sensitivity of the spin qubit to the target field by sweeping the amplitude of the magnetic field and measuring the slope of the signal $s(T)=\left(1+e^{-\chi_{n}(T)} \cos \left(\phi_{n} b\right)\right) / 2$ at the points of maximum slope (where $s=0.5$ ), as shown in Fig. 2(a). From this quantity, we extract the experimental observable $\mathcal{E}=$ $\max \left\{\partial_{b} s\right\} / \sqrt{T}$. Following Eq. (8), this experimental observable is simply related to sensitivity through the relation $\mathcal{E}=C / \eta$, where $C$ is the only calibration constant, independent of the target field strength and of the control sequence (see Appendix A 4).

In the following, we consider different time-varying target fields that mimic signals of interest and highlight the advantage of our optimal control method. The method can, however, be applied more broadly (see Ref. [59]), over the same frequency range accessible by a nonoptimized sensor, with a typical bandwidth of the sensor under pulsed control set by the pulse length and by the control-dependent decoherence time $T_{2}(\sim 2 \mathrm{kHz}-15 \mathrm{MHz})$.

\section{A. Optimized sensing of an oscillating field}

We first focus on the simple case of monochromatic sinusoidal signals $b(t)=b \cos (2 \pi \nu t+\alpha)$, with $\nu \approx$ 20-1900 kHz (see Ref. [59]). We note that measuring signals in this frequency range at a magnetic field $B \sim$ $39.4 \mathrm{mT}$ is a difficult task since the signal is obscured by the carbon-13 bath field. We start from a common pulsed dynamical decoupling sequence, the Carr-Purcell (CP) multipulse sequence, originally devised in nuclear magnetic resonance $[60,61]$, which has been demonstrated to extend the qubit's coherence [62] and has been successfully employed in sensing to measure monochromatic ac magnetic fields (see, e.g., Refs. [63-67]). CP is composed of $n$ $\pi$ pulses, equally spaced by $\tau=T / n$, which periodically flip the spin qubit. This kind of sequence is highly selective in frequency: Its filter function $Y_{n, T}(\omega)$ is indeed peaked at $\nu=1 /(2 \tau)[68]$. In the case of interest, the qubit is subject to colored noise due to a nuclear spin bath in diamond, where the main component is due to carbon-13. If the target signal has frequency close to the center of the noise spectrum, CP control may not be the best choice since the sequence achieving noise cancellation also leads to a significant attenuation of the signal to be measured.

As a warmup for the full optimization, we optimize the control over a restricted space of two parameters, the sensing time $T$ and the initial phase shift $\alpha$, with a fixed number of pulses, $n=8$. First, fixing $\alpha=0$, we find the optimal sensing time as a function of the ac frequency, as reported in Fig. 2(b). Taking into account decoherence effects appreciably modifies the optimal sensing time (purple curve), compared to the results obtained in the absence of noise sources (black curve), where the optimization routine recovers the expected analytic solutions $T_{\text {opt }}=n /(2 \nu)$. Then, we optimize both $T$ and $\alpha$. To evaluate the global convergence of the optimization, we
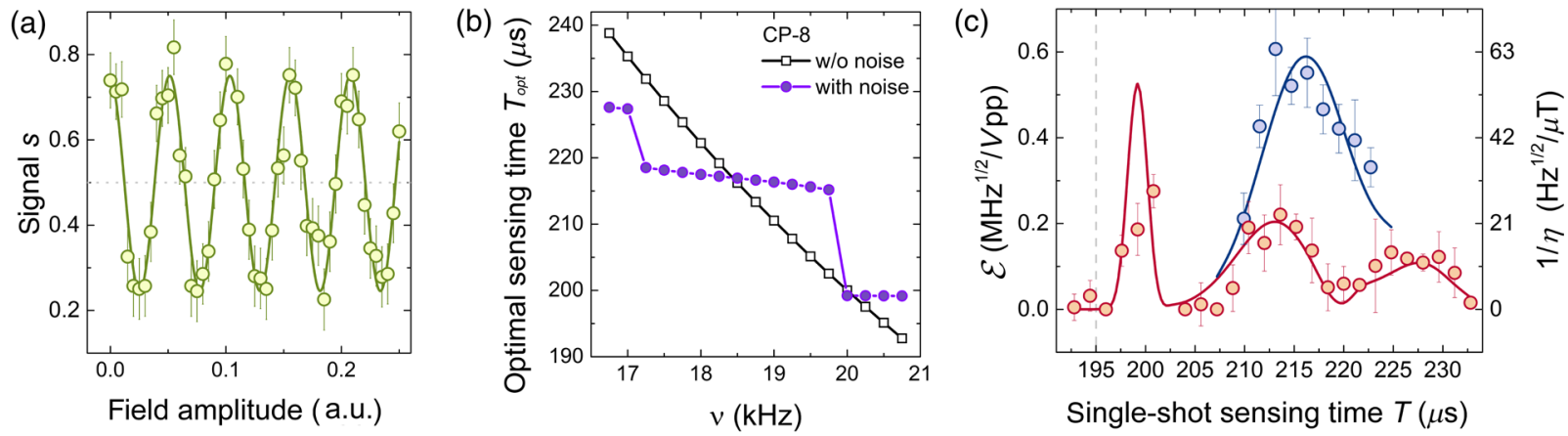

FIG. 2. Optimized sensing of monochromatic fields. (a) Experimental signal measured in the presence of a monochromatic target ac magnetic field, $b(t)=b \cos (2 \pi \nu t+\alpha)$, with $\nu=9.24 \mathrm{kHz}$ and $\alpha=0$, as a function of the target magnetic field amplitude $b$. Here, the spin sensor is controlled with a Carr-Purcell sequence of $n=4$ equidistant $\pi$ pulses. Dots are the experimental data, the curve is a cosinusoidal fit. Error bars are the statistical errors over $2 \times 10^{5}$ repeated measurements. (b) Theoretical prediction of the optimal sensing time of a Carr-Purcell sequence of $n=8$ equidistant $\pi$ pulses (CP-8), calculated for a target ac magnetic field $b(t)=b \cos (2 \pi \nu t+\alpha)$, as a function of the ac frequency $\nu$. The black empty squares are the optimized solutions of the sensing problem when neglecting the presence of the noisy environment, and the black curve represents the expected optimal time, $T_{\text {opt }}=n /(2 \nu)$, with no fitting parameters. Purple dots are optimized solutions including the noise-induced decoherence of the spin qubit (the line is a guide to the eye). (c) Inverse of sensitivity, in the presence of a cosinusoidal field $b(t)$ of frequency $\nu_{0}=20.5 \mathrm{kHz}$ under CP control, with $\alpha=0$ (in red), and with $\alpha=102^{\circ}$ (that is, an initial delay time $t_{0}=0.28 / \nu_{0}$ ) resulting from optimization (in blue). Dots are experimental value of $\mathcal{E}$ (left side vertical axis — see text), lines are theoretical $1 / \eta$ (right side vertical axis). The experimental error bars come from the slope uncertainty of the experimental signal $s$. 
have also mapped $1 / \eta$ in the two-dimensional (2D) parameter space $(T, \alpha)$. Figure 1(b) shows this map for an ac field of frequency $\nu_{0}=20.5 \mathrm{kHz}$. This allows the results of the optimization to be compared with the bruteforce approach of an extensive search in the parameter space. The full optimization of the two parameters, including noise effects, is able to find the global minimum of sensitivity (the optimized parameters are $T=216 \mu$ s and $\alpha=102^{\circ}$, corresponding to an initial delay time $t_{0}=$ $0.28 / \nu_{0}$ of the control sequence). Figure 2(c) shows some cuts of the previous $2 \mathrm{D}$ map as a function of $T$, with $\alpha=0$ (red solid line) and with $\alpha=102^{\circ}$ (the optimal value resulting from the numerical search, blue line). Using Eq. (8), we also calculate the experimental observable $\mathcal{E}=$ $C / \eta$ [left side, vertical axis in Fig. 2(c)], which can be directly compared with the experimental findings at fixed $\alpha=0$ (red dots) and with $\alpha=102^{\circ}$ (blue dots). We find good agreement of the experiments with the results of the optimization. We also remark that even in the simple case of one-parameter optimization, including the noise effects yields a different optimal sensing time than what was calculated in the absence of noise $\left[T=n /\left(2 \nu_{0}\right)\right.$, gray vertical dashed line], and this is also reflected in the observed experimental peak of $\mathcal{E}$ vs $T$.

\section{B. Optimized sensing of multitone ac signals}

We now tackle the more complex task of measuring arbitrary time-dependent signals. We consider multitone magnetic fields, in the form $b(t)=b \sum_{i}^{m} w_{i} \cos \left(2 \pi \nu_{i} t+\alpha_{i}\right)$, where $m$ is the number of Fourier components, $b_{i}=b w_{i}$ their amplitudes (with $\sum_{i} w_{i}=1$ ), $\nu_{i}$ their frequencies, and $\alpha_{i}$ the initial phases. We employ our optimization tool to engineer optimal control sequences of nonequidistant $\pi$ pulses that may extract information from multitone target signals while refocusing spin dephasing better than common dynamical decoupling solutions.

As already mentioned, common multipulse control sequences like CP are, in general, highly selective in frequency. For this reason, these sequences may exhibit suboptimal performances when probing a multitone target field due to attenuation of some frequency components. In addition, increasing the interrogation time to enable a larger phase accumulation, thus improving measurement sensitivity, also further narrows the width of the filter function $Y_{n, T}$ as $\sim 1 /(T)$ [3]. When the magnetometry task consists in measuring the signal amplitude of a spectrally characterized source, as we assume here, CP can mostly collect information about just one frequency component, for any fixed sensing time. When fixing the number of pulses $n$ and sweeping the total time $T=n \tau$, the phase accumulated by the spin qubit sensor under $\mathrm{CP}$ control reflects the spectral composition of the signal, showing peaks at times $\tau_{i}=1 /\left(2 \nu_{i}\right)$. This is exemplified in Fig. 3(b), where we consider a field made of $m=3$ Fourier components under a $\mathrm{CP}$ train of $n=50$ pulses (green solid line). However, the sensor's decoherence influences the final sensitivity by (a)

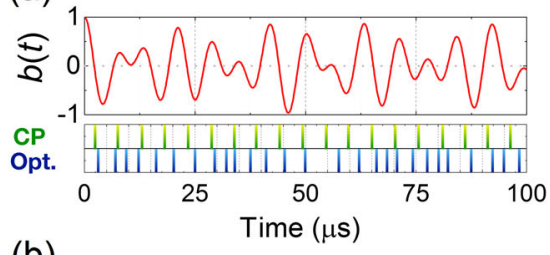

(b)

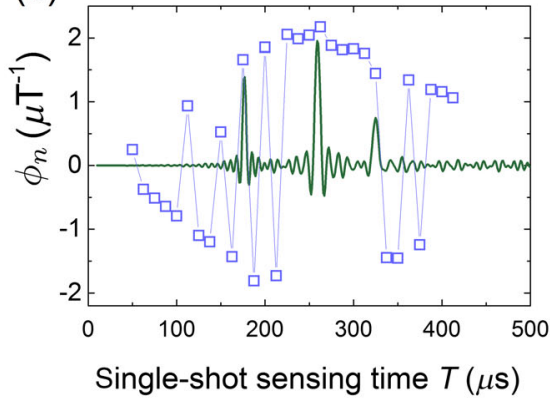

(c)

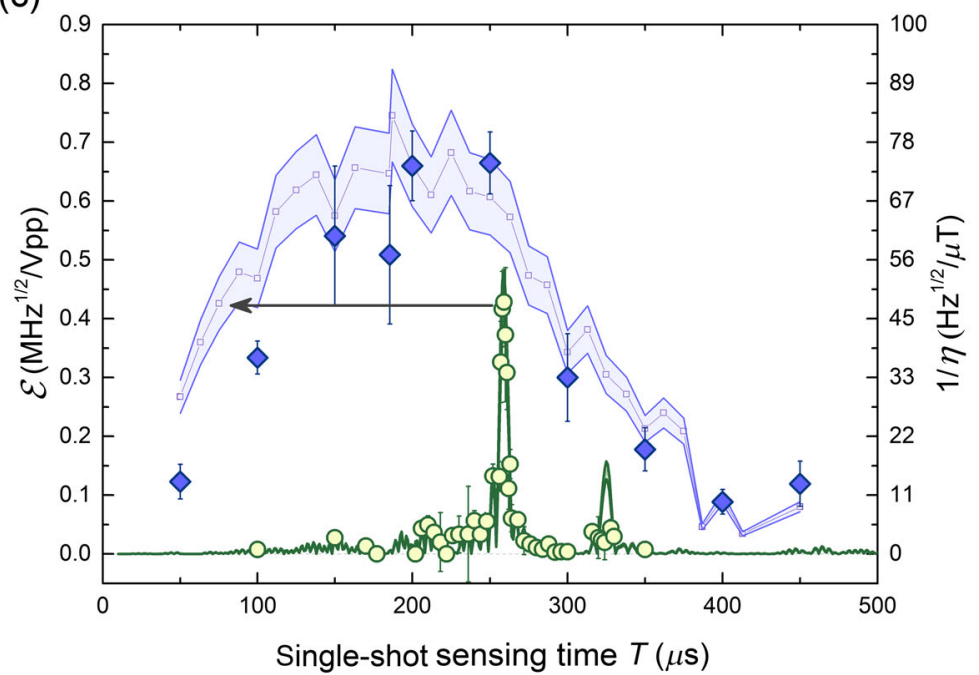

FIG. 3. Optimized sensing of multitone ac fields. (a) Upper panel: Sample multitone target field, $b(t)=b \sum_{i}^{3} w_{i} \cos \left(2 \pi \nu_{i} t+\alpha_{i}\right)$, with $\alpha_{i}=0$, frequencies $\nu_{i}=(77 ; 96 ; 141) \mathrm{kHz}$, and amplitudes $w_{i}=(0.12 ; 0.43 ; 0.45)$, respectively. Bottom panel: In green, position of the first $19 \pi$ pulses of a Carr-Purcell sequence of 50 equidistant $\pi$ pulses (CP-50) with optimized sensing time ( $T=260 \mu$ s); in blue, position of the first $27 \pi$ pulses of an optimal control sequence of 50 non-equally-distributed $\pi$ pulses with optimized time intervals and optimized initial phase $\left(T=187 \mu \mathrm{s}, \alpha_{i}=0.3\right)$. (b) Phase $\phi_{n}(T)=\varphi_{n}(T) / b$ accumulated by the spin qubit sensor during the sensing time $T$ in the presence of the field $b(t)$, under a control field of $n=50 \pi$ pulses, in the cases of CP-50 (solid line) and optimized control (blue squares). (c) Experimentally measured $\mathcal{E}=C / \eta$ in the presence of the field $b(t)$ under CP-50 (dots) and optimized control (diamonds). The curves represent the theoretical prediction for $\mathcal{E}$, for $\mathrm{CP}-50$ (solid green line) and optimized control (blue line), respectively, obtained by rescaling $1 / \eta$ (right-hand side, vertical scale) with the unique factor $C$. The shaded area takes into account the experimental uncertainty due to $C$ (see Appendix A 4). 
suppressing the response of one of the three frequency components, as shown both in theory and in experiment [green solid line and yellow dots in Fig. 3(c)].

For this kind of scenario, optimal control strategies offer a key advantage. Optimal control can indeed be exploited to find optimal distributions of the $\pi$-pulse positions. Sequences of non-equally distributed pulse spacings, devised by means of analytical models, have been indeed demonstrated to correct for selectivity of CP in certain cases [69-72], also for different experimental platforms [50]. In the case of multitone ac signals to be measured, such sequences enable to simultaneously collect signal from all the various frequency components thus achieving a faster phase accumulation. The results of optimization for the multitone field considered above are shown in Fig. 3 [73]. For the optimization, we keep the number of pulses fixed to $n=50$, and optimize all the $\pi$-pulse positions and the initial phase $\left(\alpha_{i}=\alpha\right)$, in all 51 free parameters. We impose the time intervals around to each $\pi$ pulse to be symmetric with respect to the pulse position in order to ensure cancellation of static noise and better refocusing of low-frequency noise (see Appendix A 4). As shown in Fig. 3(b), the optimization method leads to a remarkable improvement in the accumulated phase per unit field amplitude $\phi_{n}$ (blue squares) compared to CP (green line), over an extremely wide range of sensing times. The overall-optimal control sequence (obtained with sensing time $T=187 \mu$ s and phase shift $\alpha=0.3$ ) realizes a sensitivity $\eta_{\mathrm{opt}}^{\text {best }}=12 \mathrm{nT} / \sqrt{\mathrm{Hz}}$. Since each of our sensing experiments is typically obtained by averaging over $N=2 \times 10^{5}$ measurement shots, the optimized control sequence enables the measurement of a local field of $2 \mathrm{nT}$. The improvement in sensitivity is more than 2 orders of magnitude compared to the sensitivity of $\mathrm{CP}\left(\eta_{\mathrm{CP}}=8.3 \mu \mathrm{T} / \sqrt{\mathrm{Hz}}\right)$ at the same sensing time. We remark that the best sensitivity obtained with CP control is still a factor of 1.75 worse than the best sensitivity achieved with the optimized control $\left(\eta_{\mathrm{CP}}^{\text {best }}=21 \mathrm{nT} / \sqrt{\mathrm{Hz}}\right)$ and with an acquisition time $(T=$ $260 \mu \mathrm{s})$ that is $40 \%$ longer than the optimal sequence. In addition, optimized control is able to achieve the same $\eta_{\mathrm{CP}}^{\text {best }} 3$ times faster than CP [ $T=75 \mu \mathrm{s}$, compared to $T=260 \mu \mathrm{s}$; see black arrow in Fig. 3(c)]. Thus, optimal control is able to attain a remarkable enhancement of sensitivity compared to standard control when measuring multitone ac fields at fixed interrogation time. Besides, optimal control makes it possible to speed up the measurement while maintaining the same sensitivity of standard control, e.g., when measuring target signals with inherently short coherence times or in the presence of fast experimental drifts that limit the available coherent single-shot measurement time.

\section{Optimized sensing of trains of magnetic impulses}

We have applied optimal control to the different scenarios where the target magnetic field is a train of impulses. This is, in general, the case of the temporal shape of electric and magnetic fields associated with cardiac, neural, and nervous activities of human and animal organs [34-37]. For this kind of application, the NV sensors may offer the remarkable advantages of subcellular spatial resolution, in addition to high sensitivity and biocompatibility [74].

As illustrative models for these biological applications, we consider a train of Gaussian-shaped impulses. The target field is thus of the general form, shown in Fig. 4(a),

$$
b(t)=b \sum_{i=0}^{m_{r}} e^{-\left[(t-i \Delta t)^{2} / 2 \sigma^{2}\right]},
$$

where $1 / \Delta t$ is the repetition rate and $m_{r}$ is the number of repetitions, with $m_{r} \Delta t \gg T$. In this case, standard dynamical decoupling may be underperforming since the target signal $b(t)$ is positive definite in the whole temporal domain; thus, the product $y_{n}(t) b(t)$ may be alternately positive and negative, reducing the accumulation of a useful phase [see Eq. (2)]. In other words, each time a $\pi$ pulse reverses the spin dynamics, it can partially cancel not only the effect of unwanted noise but also the phase associated with the field to be measured.

Figures 4(b)-4(i) compare the results of CP control (green-colored curves) and optimal control (in blue), when varying the width $\sigma$ of the target Gaussian pulse train and its repetition rate $1 / \Delta t$ [59]. In this case as well, we evaluate the effect of control sequences made of $n=50 \pi$ pulses. In the optimization, all the time intervals between the control pulses, symmetrized around the $\pi$-pulse positions, are free parameters. The left panels represent the modulus of the phase accumulated by the spin qubit sensor per unit of the target magnetic field amplitude $\left|\phi_{n}\right|$, whereas the right panels represent the inverse of sensitivity $1 / \eta$, as a function of the sensing time $T$.

Optimal control outperforms $\mathrm{CP}$ in accumulating a useful phase due to $b(t)$ over a large sensing-time range. Both CP and optimal control lead to their largest phase accumulation when $T \simeq n \Delta t / 2$, where they give similar results in $\phi_{n}$. This condition corresponds to having couples of $\pi$ pulses located in each "empty" time window between two Gaussian pulses of the target field, albeit optimal control corrects, in a nontrivial way, the distribution of $\pi$-pulse positions to minimize $\eta$, as represented in Fig. 4(a) (CP, green vertical bars; optimal control, blue vertical bars). Thus, the $\pi$ pulses partially reverse the spin qubit dynamics due to undesired noise but do not cancel the phase due to the target field $b(t)$.

We note that, even when the phases accumulated with $\mathrm{CP}$ and with optimal control are comparable, optimal control compensates better than $\mathrm{CP}$ for decoherence, leading to better overall sensitivity. While here we did not explore this result further, it seems to indicate that numerically optimized sequences might also be useful for other quantum information tasks, such as building a robust memory. Figures 4(e), 4(g), and 4(i) show that optimal control of the spin qubit improves its sensitivity to Gaussian 


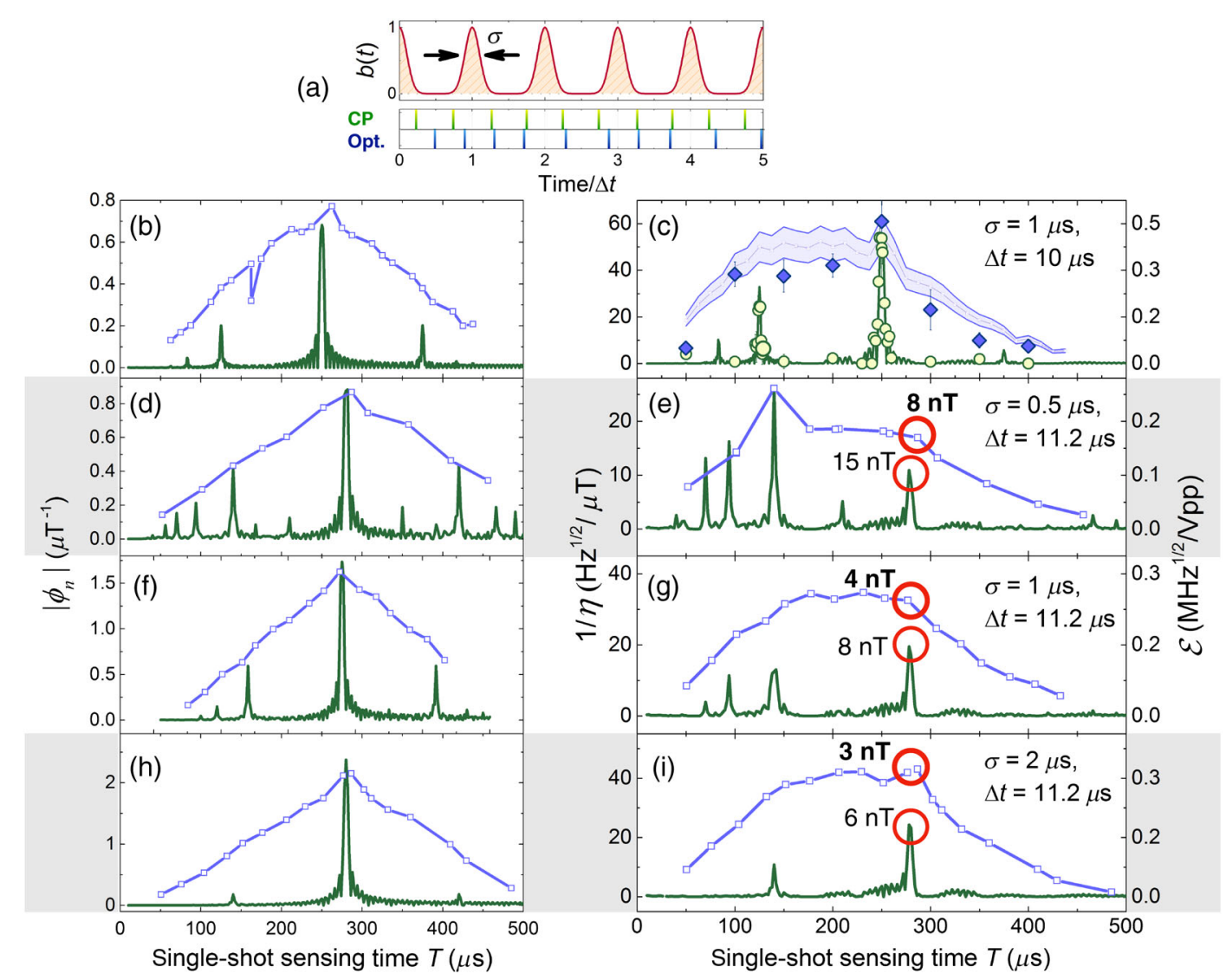

FIG. 4. Optimal sensing of Gaussian impulses. (a) Upper panel: Target signal made of a train of Gaussian impulses of width $\sigma$ and repetition rate $1 / \Delta t$. Bottom panel: Position of the first 10 pulses of a CP-50, with total sensing time $T=280 \mu$ s optimized to sense a train of Gaussian impulses with $\sigma=2 \mu \mathrm{s}, \Delta t=11.2 \mu \mathrm{s}$ (in green), and optimal position of the first 10 pulses of a control sequence of 50 pulses (in blue), optimized to sense the same target field (50 optimization parameters). (b)-(i) Modulus of the phase accumulated by the spin qubit sensor in the presence of the target field (left panels), and the inverse of sensitivity $1 / \eta$ (right panels), as a function of the sensing time $T$, under CP-50 control (green solid curves) and under optimized control (blue lines with squares) with bounds $\tau \in(0.6-10) \mu$ s (see Appendix A 3). (c) Measurement of the experimental observable $\mathcal{E}$ as resulting from $\mathrm{CP}$ experiments (yellow dots) and from optimized control (blue diamonds), scaling as indicated on the right-hand side, vertical axis. The target field parameters of panels (b)-(i) are as follows: (b,c) $\sigma=1.0 \mu \mathrm{s}, \Delta t=10 \mu \mathrm{s}$; (d,e) $\sigma=0.5 \mu \mathrm{s}, \Delta t=11.2 \mu \mathrm{s}$; (f,g) $\sigma=1.0 \mu \mathrm{s}, \Delta t=11.2 \mu \mathrm{s}$; (h,i) $\sigma=2.0 \mu \mathrm{s}, \Delta t=11.2 \mu \mathrm{s}$.

multipulse signals up to a factor of 2 , enabling the measurement of multipulse magnetic fields down to 3 nT. As shown in Fig. 4(c), the measurement of the experimental observable $\mathcal{E}=C / \eta$ confirms the theoretical prediction of sensitivity both for the $\mathrm{CP}$ control (yellow dots) and for optimal control (blue diamonds).

\section{DISCUSSION}

We have devised a versatile and robust method of optimal control for quantum metrology with one qubit, and we have applied this optimal control method to the measurement of weak time-varying magnetic fields with a NV spin sensor.

The key insight of our optimal control strategy is the introduction of an unconventional optimization metric, the qubit sensor's sensitivity. The minimization of sensitivity is made by searching the optimal control field that realizes the optimal compromise between useful accumulation of the spin phase due to the external field to be measured and noise refocusing. The developed optimization algorithm offers the advantage of fast convergence and simplicity. We have further investigated the robustness of this method for different kinds of real target fields. Optimal control outperforms standard dynamical decoupling in different scenarios, ranging from multicomponent ac target fields in a wide frequency range of the radio-frequency (rf) domain, to trains of impulses, which are illustrative examples of the typical shape of the electromagnetic field of interest in biology and physiology.

In the cases investigated, optimal control enables larger phase accumulation over wide sensing-time windows, as well as better cancellation of the effect of external noise on the spin dynamics. Sensitivity of the qubit sensor under optimized control shows an improvement by up to a factor of 2, enabling the measurement of a pulsed magnetic field down to amplitudes of $2 \mathrm{nT}$. The comparison of $1 / \eta$ with 
the experimental observable $\mathcal{E}$ demonstrates the reliability of this optimal control method applied to the NV spin sensor.

Beyond the results obtained in exemplary situations, our novel method is one of the first extensions of optimal control methods to quantum sensing. This application raises novel challenges and opportunities, in particular, related to the need for new metrics for optimization, as well as the challenge to include nonunitary evolution in the numerical optimization. We underline that our optimization method can be extended to a larger multidimensional space of parameters; for instance, one can optimize the number of $\pi$ pulses that flip the spin qubit during the sensing time, according to the target signal to be measured. Moreover, while we always considered control sequences given by a series of $\pi$ pulses, our scheme can also be generalized to other control strategies of the NV spin qubit.

Our strategy can be useful for metrology in the face of more and more demanding requirements for the NV spin qubit to sense weak time-varying electric and magnetic fields in noisy environments, with the sensing task aimed at measuring their amplitude, e.g., for detecting the number of spins in small electronic or nuclear ensembles [75-77], revealing excitations in nanostructured antiferromagnetic or multiferroic materials [78], or measuring biological activity at the nanoscale [37]. Adaptive strategies could also be used to extend this scheme to fields with an unknown temporal profile. Moreover, the method can also be applied to other physical platforms, such as ultracold atoms or trapped ions. Furthermore, the demonstrated enhanced protection of the spin qubit against noise-induced decoherence also makes optimal control a strategic tool for building memories in solid-state systems.

\section{ACKNOWLEDGMENTS}

We especially thank M. Inguscio for enthusiastic support, S. Hérnadez Gómez for critical reading, and the LENS Quantum Gases group for useful discussions. This work was supported by European Research Council through Starting Grant Q-SEnS2 (No. 337135).

\section{APPENDIX: METHODS}

\section{Experimental setup}

The host diamond crystal used in this study is a monocrystalline electronic-grade sample (Element Six), grown via chemical vapor deposition, with a natural $1.1 \%$ abundance of ${ }^{13} \mathrm{C}$ impurities and ${ }^{14} \mathrm{~N}$ concentration $\ll 5 \mathrm{ppb}$. All of the experiments have been performed on a single, negatively charged NV center, located at about $13.5 \mu \mathrm{m}$ below the diamond surface.

A permanent $\mathrm{NdFeB}$ magnet produces an external static magnetic field, $B=39.4 \mathrm{mT}$, aligned along the symmetry axis of the NV center ( $\hat{z}$ axis). The field lifts the degeneracy of the $m_{s}=+1$ and $m_{s}=-1$ energy levels.
We exploit a home-built confocal microscope to focus a 532-nm laser beam on the defect and to collect the emitted fluorescence [39]. The laser excitation initializes the NV spin in the $m_{s}=0$ state. We perform the spin-state readout after manipulation by measuring the fluorescence photon count rate (about $5 \times 10^{4} \mathrm{cps}$, with contrast of about $30 \%$ ) with a single photon detector. The measured fluorescence is then normalized to the difference between the fluorescence intensities of the pure states $m_{s}=0$ and $m_{s}=-1$ to obtain the signal $s$, that is, the probability of the electron spin state to be in the state $m_{s}=-1$. Each data point is averaged over $N=2 \times 10^{5}$ repetitions to increase the signal-to-noise ratio.

Control of the NV spin dynamics is obtained by irradiating the defect with microwave pulses. We routinely use mw $\pi$ pulses that repeatedly flip the spin, in order to periodically reverse its temporal evolution and refocus the noise effect. The mw pulse trains are applied through a $60-\mu \mathrm{m}$ thin copper wire that works as an antenna. We exploit the same wire to deliver time-varying magnetic fields in the rf range generated by an arbitrary waveform generator. These rf signals are the target magnetic fields to be measured by the NV. Using two different terminals of the wire, we can simultaneously apply both mw and rf fields.

\section{Measuring the sensor noise spectrum}

Our optimal control strategy depends on knowledge of the coherence function $\chi_{n}(T)$. As shown in Eq. (4), $\chi_{n}(T)$ depends on both the noise spectrum and the frequency filter $Y_{n, T}(\omega)$ given by the specific sensing sequence. In order to compute the sensitivity $\eta$ [Eq. (8)], the optimization algorithm calculates the value of $\chi_{n}(T)$ for different trial sequences; thus, it needs the noise spectral density $S(\omega)$ as an input. Various methods to measure the noise spectrum have been suggested in the literature [48,53,79-83]. Here, we follow the procedure described in Ref. [79].

The filter function $Y_{n, T}(\omega)=\left|y_{n}(\omega, T)\right|^{2}$, where $y_{n}(\omega, T)$ represents the Fourier transform of the modulation function $y_{n}(t)$, has a simple form for periodic sequences. In the limit of large pulse numbers, it can be approximated by a delta function at the angular frequency $\pi / \tau$, where $\tau$ is the pulse spacing. Then, the coherence signal decays as $s(t) \sim$ $e^{-t / T_{2}^{\mathrm{CP}}(\tau)}$, where $T_{2}^{\mathrm{CP}}(\tau)$ is a coherence time directly related to the noise spectral density via [79]

$$
\frac{1}{T_{2}^{\mathrm{CP}}(\tau)} \simeq \frac{4}{\pi^{2}} S(\pi / \tau)
$$

For each value of $\tau$ (which sets the noise frequency that we are considering), we measured the signal decay as a function of the number of pulses, obtaining $T_{2}^{\mathrm{CP}}(\tau)$. By varying the time $\tau$ between mw $\pi$ pulses, we can extract the main frequency components of the noise spectral density using Eq. (A1). The spectrum was finally obtained by fitting the raw data with a sum of Gaussian functions. 
The experimental spectrum $S(\omega)$ obtained for sequences with different numbers of pulses showed some variation. Thus, we further refine $S(\omega)$ by fitting the signal $s(T)$ measured after a CP decoupling sequence as in Ref. [46] (see, e.g., Fig. 5). This procedure is aimed at correcting $S(\omega)$ for sequence-dependent noise and control imperfection, e.g., due to the finite $\pi$-pulse duration [84], which are not taken into account in our model. We finally find a noise spectrum that, for a fixed number of pulses $n$, is completely independent of the timing at which each $\pi$ pulse occurs; thus, it can be used for sequences that are very different from CP in the optimization procedure.

The experimental signal $s(T)$ exhibits periodic collapses due to the nuclear spin bath, with additional dips below 0.5 (see Fig. 5) that are a signature of a quantum environment, more precisely, of a hyperfine interaction between the NV electronic spin and isolated nearby nuclear spins [65,85]. As the latter interactions give rise to unitary evolution of the $\mathrm{NV}$, we exclude them from our noise model. This allows us to use Eq. (4), which cannot take them into account but provides a speedup of the numerical optimization. We emphasize that good agreement between predicted and measured sensitivity (also for $150 \mu \mathrm{s}<T<200 \mu \mathrm{s}$, where we see a population of less than 0.5 ; see Fig. 3 and Ref. [59]) is proof of the robustness of the model to the effects of weak couplings between NV and isolated spins, as well as to imperfections in the empirical function $\chi_{n}(T)$. Including pure quantum noise effects would be an interesting technical extension of the present method.

\section{Optimization algorithm}

The core of our optimal control technique for sensing is an optimization algorithm that minimizes the sensitivity as a function of the parameters of the control function, e.g.,

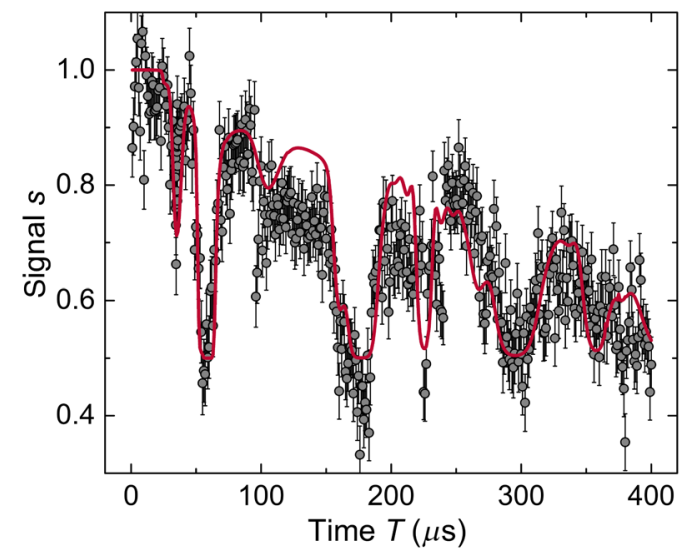

FIG. 5. NV spin coherence under the CP sequence. Measured signal $s(T)$ (gray dots) after a CP decoupling sequence with $n=50$, as a function of time $T=n \tau$. Here, the sensor is not irradiated with any external target field $b(t)$ to be measured. The solid red line is a fit of the signal based on a classical model of the bath (see text). total sensing time, phase of the ac field, and time intervals between $\pi$ pulses.

We use a MATLAB routine based on the simplex minimization algorithm to achieve global optimization of the figure-of-merit, the sensitivity $\eta$. The two main ingredients of this quantity are the electron spin phase $\varphi_{n}(T)$ and the coherence function $\chi_{n}(T)$. We consider pulsed control sequences described by the $\pi$-pulse times $\left\{t_{j}\right\}$. For any time-varying external magnetic field $b(t)=b f(t)$ to be measured, we can define $F(t)=(1 / t) \int_{0}^{t} f\left(t^{\prime}\right) d t^{\prime}$, the integral of the magnetic field (known) temporal profile $f(t)$. The phase $\varphi_{n}=b \phi_{n}$ acquired by the NV qubit can then be calculated for any given control sequence as

$$
\phi_{n}=(-1)^{n+1} F(T) T-2 \sum_{j=0}^{n+1}(-1)^{j} F\left(t_{j}\right) t_{j} .
$$

The coherence $\chi_{n}(T)$ is instead obtained from the experimentally measured spectrum via Eq. (4). From $\chi_{n}(T)$ and $\phi_{n}(T)$, we can calculate $\eta$ for each trial sequence according to Eq. (8).

In order to verify the global convergence of the optimization algorithm, we tested different initial guesses and found the same optimized parameters for a given ac target field. In most cases, we used a constrained search, by setting bounds for each parameter or constraining the overall result, for instance, to keep the total time $T$ constant.

We note that our procedure is quite general and could be applied to a broad range of sensing scenarios. To demonstrate its reliability, in this work we considered a few exemplary target fields and related control models, varying, e.g., the number of parameters tackled by the optimization algorithm. We first considered ac fields with single or multiple frequencies, and we started optimizing $\eta$ as a function of total time $T$ and the ac field phase $\alpha$, while fixing the number of pulses $(n=8)$ and setting $\tau=T / n$ for all time intervals between the $\pi$ pulses. We then proceeded to allow more flexibility in the optimization by varying the duration of each time interval between $\pi$ pulses, starting from an initial guess given by a periodic (CP) sequence with $n=50$. We optimized the time intervals by keeping the time symmetric around each pulse, as shown in Fig. 6. Including also the optimization of the phase of the

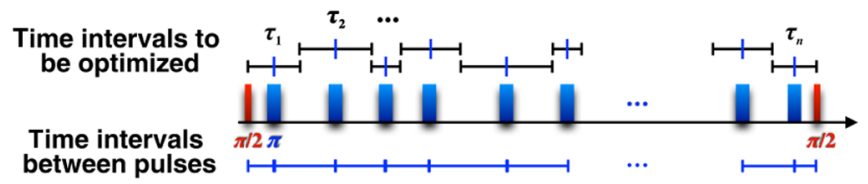

FIG. 6. Time interval $\tau_{j}$ engineering. Optimization scheme of the $n$ time intervals $\tau_{j}$ of a measurement sequence with $n$ pulses. Here, $\tau_{j}=\left(t_{j}+t_{j+1}\right) / 2$, where $t_{j}$ are the $n+1$ time intervals between the $\pi$ pulses, with $j=0, \ldots, n$, and $t_{0}=t_{n+1}=0$. 
multitone field, or equivalently, the initial time of the measurement sequence, this optimization manages 51 free parameters. We performed different optimization runs as a function of the total measurement time, keeping $T$ constant in each of them. The only additional constraint that we imposed was to force the times $\tau_{j}$ between different $\pi$ pulses to be longer than about 10 times the $\pi$ pulse duration, which in our case means $\tau_{j}>600 \mathrm{~ns}$. This restriction was to ensure that no mw pulses would be very close to each other, as that would have resulted in the $\pi$ pulses canceling each other, giving an effective sequence with a different $n$.

\section{Comparison between optimal control theory and experiment}

To experimentally validate the optimal control, we compare the optimized sensitivity $\eta$ with the corresponding measured quantity. However, since we do not have an independent measure of the local amplitude of the magnetic field at the position of the NV center, in the experiment we measure $\mathcal{E}=\max \left\{\partial_{b} s\right\} / \sqrt{T}=C / \eta$, where $s$ is the normalized signal and $C$ represents a conversion factor between the generated rf field amplitude and the unknown magnetic field at the defect. As $C$ does not depend on the control sequence, it can be evaluated once and then used for all the control scenarios considered in the paper.

In particular, we estimate $C$ from the experimental results for $\mathrm{CP}$ sequences. We experimentally evaluate $\mathcal{E}(T)$ as a function of the sequence total time $T$ and fit the curve to extract the maximum $\mathcal{E}_{C P_{M}}$. Similarly, we evaluate the theoretical value of $\eta$ and obtain its minimum $\eta_{C P_{M}}$. We then define $C$ as the product $C=\mathcal{E}_{C P_{M}} \eta_{C P_{M}}$. This procedure allows us not only to define $C$ but also to estimate its uncertainty $\Delta C$, from the fit error. We can then compare the (inverse) experimental sensitivity $\mathcal{E}_{i}$ and the theoretical sensitivity $\eta_{i}$ for each control sequence by rescaling the theoretical sensitivity by $C$.

Finally, we investigate the effect of the finite $\mathrm{mw}$ pulse duration. Considering the case of a Gaussianshaped train of magnetic impulses under a $\mathrm{CP}$ control sequence, we calculate phase accumulation and sensitivity when excluding from the spin evolution the time intervals where the $\pi$ pulses occurs, finding the same theoretical values of $\eta$, for all the considered total sensing times $T$. We underline that this procedure does not correct the model for the contribution to $\chi_{n}(T)$ given by mw pulse imperfections or finite duration [84], but it confirms that the pulses can be considered instantaneous in our picture for the accumulated spin phase $\varphi_{n}(T)$; we verified that this approximation is valid up to $\pi_{\mathrm{mw}} \leq 0.5 \mu \mathrm{s}$ for $n=50$ and $T \sim 250 \mu$ s and is very effective for pulses duration $\pi_{\mathrm{mw}}=56 \mathrm{~ns}$ and $T \geq 50 \mu$ s.
[1] M. A. Nielsen and I. L. Chuang, Quantum Computation and Quantum Information (Cambridge University Press, Cambridge, 2000).

[2] V. Giovannetti, S. Lloyd, and L. Maccone, Advances in Quantum Metrology, Nat. Photonics 5, 222 (2011).

[3] C. L. Degen, F. Reinhard, and P. Cappellaro, Quantum Sensing, Rev. Mod. Phys. 89, 035002 (2017).

[4] D. J. Wineland, D. Leibfried, M. D. Barrett, A. Ben-Kish, J. C. Bergquist, R. B. Blakestad, J. J. Bollinger, J. Britton, J.hiaverini, B. Demarco, D. Hume, W. M. Itano, M. Jensen, J. D. Jost, E. Knill, J. Koelemeji, C. Langer, W. Oskay, R. Ozeri, R. Reichle et al., Quantum Control, Quantum Information Processing, and Quantum-Limited Metrology with Trapped Ions, in Laser Spectroscopy (World Scientific, Singapore, 2005).

[5] N. Timoney, V. Elman, S. Glaser, C. Weiss, M. Johanning, W. Neuhauser, and C. Wunderlich, Error-Resistant SingleQubit Gates with Trapped Ions, Phys. Rev. A 77, 052334 (2008).

[6] P. S. Jessen, D. L. Haycock, G. Klose, G. A. Smith, I. H. Deutsch, and G. K. Brennen, Quantum Control and Information Processing in Optical Lattices, Quantum Inf. Comput. 1, 20 (2001).

[7] S. Rosi, A. Bernard, N. Fabbri, L. Fallani, C. Fort, M. Inguscio, T. Calarco, and S. Montangero, Fast Closed-Loop Optimal Control of Ultracold Atoms in an Optical Lattice, Phys. Rev. A 88, 021601 (2013).

[8] M. D. Reed, L. DiCarlo, S. E. Nigg, L. Sun, L. Frunzio, S. M. Girvin, and R. J. Schoelkopf, Realization of ThreeQubit Quantum Error Correction with Superconducting Circuits, Nature (London) 482, 382 (2012).

[9] A. Spörl, T. Schulte-Herbrüggen, S. J. Glaser, V. Bergholm, M. J. Storcz, J. Ferber, and F. K. Wilhelm, Optimal Control of Coupled Josephson Qubits, Phys. Rev. A 75, 012302 (2007).

[10] D. Cory, R. Laflamme, E. Knill, L. Viola, T. Havel, N. Boulant, G. Boutis, E. Fortunato, S. Lloyd, R. Martinez, C. Negrevergne, M. Pravia, Y. Sharf, G. Teklemariam, Y. Weinstein, and W. Zurek, NMR Based Quantum Information Processing: Achievements and Prospects, Fortschr. Phys. 48, 875 (2000).

[11] J. Zhang, D. Gangloff, O. Moussa, and R. Laflamme, Experimental Quantum Error Correction with High Fidelity, Phys. Rev. A 84, 034303 (2011).

[12] J. Wrachtrup and F. Jelezko, Processing Quantum Information in Diamond, J. Phys. Condens. Matter 18, S807 (2006).

[13] G. Waldherr, Y. Wang, S. Zaiser, M. Jamali, T. SchulteHerbruggen, H. Abe, T. Ohshima, J. Isoya, J. F. Du, P. Neumann, and J. Wrachtrup, Quantum Error Correction in a Solid-State Hybrid Spin Register, Nature (London) 506, 204 (2014).

[14] D. D'Alessandro, Introduction to Quantum Control and Dynamics (Taylor \& Francis, London, 2007).

[15] S. J. Glaser, U. Boscain, T. Calarco, C.P. Koch, W. Koeckenberger, R. Kosloff, I. Kuprov, B. Luy, S. Schirmer, T. Schulte-Herbrueggen, D. Sugny, and F. K. Wilhelm, Training Schrödinger's Cat: Quantum Optimal Control, Eur. Phys. J. D 69, 279 (2015). 
[16] E. M. Fortunato, M. A. Pravia, N. Boulant, G. Teklemariam, T. F. Havel, and D. G. Cory, Design of Strongly Modulating Pulses to Implement Precise Effective Hamiltonians for Quantum Information Processing, J. Chem. Phys. 116, 7599 (2002).

[17] N. Khaneja, T. Reiss, C. Kehlet, T. Schulte-Herbuggen, and S. Glaser, Optimal Control of Coupled Spin Dynamics: Design of NMR Pulse Sequences by Gradient Ascent Algorithms, J. Magn. Reson. 172, 296 (2005).

[18] T. Caneva, T. Calarco, and S. Montangero, Chopped Random-Basis Quantum Optimization, Phys. Rev. A 84, 022326 (2011).

[19] S. Machnes, U. Sander, S. J. Glaser, P. de Fouquières, A. Gruslys, S. Schirmer, and T. Schulte-Herbrüggen, Comparing, Optimizing, and Benchmarking Quantum-Control Algorithms in a Unifying Programming Framework, Phys. Rev. A 84, 022305 (2011).

[20] G. Ciaramella, A. Borzì, G. Dirr, and D. Wachsmuth, Newton Methods for the Optimal Control of Closed Quantum Spin Systems, SIAM J. Sci. Comput. 37, A319 (2015).

[21] C. A. Ryan, J. S. Hodges, and D. G. Cory, Robust Decoupling Techniques to Extend Quantum Coherence in Diamond, Phys. Rev. Lett. 105, 200402 (2010).

[22] J. Scheuer, X. Kong, R. S. Said, J. Chen, A. Kurz, L. Marseglia, J. Du, P. R. Hemmer, S. Montangero, T. Calarco, B. Naydenov, and F. Jelezko, Precise Qubit Control Beyond the Rotating Wave Approximation, New J. Phys. 16, 093022 (2014).

[23] D. J. Tannor and S. A. Rice, Control of Selectivity of Chemical Reaction via Control of Wave Packet Evolution, J. Chem. Phys. 83, 5013 (1985).

[24] P. Brumer and M. Shapiro, Laser Control of Molecular Processes, Annu. Rev. Phys. Chem. 43, 257 (1992).

[25] T. C. Weinacht, J. Ahn, and P. H. Bucksbaum, Controlling the Shape of a Quantum Wavefunction, Nature (London) 397, 233 (1999).

[26] S. Machnes, M. B. Plenio, B. Reznik, A. M. Steane, and A. Retzker, Superfast Laser Cooling, Phys. Rev. Lett. 104, 183001 (2010).

[27] A. Rahmani, T. Kitagawa, E. Demler, and C. Chamon, Cooling through Optimal Control of Quantum Evolution, Phys. Rev. A 87, 043607 (2013).

[28] Z. Tošner, T. Vosegaard, C. Kehlet, N. Khaneja, S. J. Glaser, and N. C. Nielsen, Optimal Control in NMR Spectroscopy: Numerical Implementation in SIMPSON, J. Magn. Reson. 197, 120 (2009).

[29] P. Doria, T. Calarco, and S. Montangero, Optimal Control Technique for Many-Body Quantum Dynamics, Phys. Rev. Lett. 106, 190501 (2011).

[30] S. van Frank, M. Bonneau, J. Schmiedmayer, S. Hild, C. Gross, M. Cheneau, I. Bloch, T. Pichler, A. Negretti, T. Calarco, and S. Montangero, Optimal Control of Complex Atomic Quantum Systems, Sci. Rep. 6, 34187 (2016).

[31] T. Häberle, D. Schmid-Lorch, K. Karrai, F. Reinhard, and J. Wrachtrup, High-Dynamic-Range Imaging of Nanoscale Magnetic Fields Using Optimal Control of a Single Qubit, Phys. Rev. Lett. 111, 170801 (2013).

[32] T. Nöbauer, A. Angerer, B. Bartels, M. Trupke, S. Rotter, J. Schmiedmayer, F. Mintert, and J. Majer, Smooth Optimal
Quantum Control for Robust Solid-State Spin Magnetometry, Phys. Rev. Lett. 115, 190801 (2015).

[33] S. Pang and A. N. Jordan, Optimal Adaptive Control for Quantum Metrology with Time-Dependent Hamiltonians, Nat. Commun. 8, 14695 (2017).

[34] J. Wikswo, J. Barach, and J. Freeman, Magnetic Field of a Nerve Impulse: First Measurements, Science 208, 53 (1980).

[35] G. Bison, N. Castagna, A. Hofer, P. Knowles, J.-L. Schenker, M. Kasprzak, H. Saudan, and A. Weis, A Room Temperature 19-Channel Magnetic Field Mapping Device for Cardiac Signals, Appl. Phys. Lett. 95, 173701 (2009).

[36] K. Jensen, R. Budvytyte, R. A. Thomas, T. Wang, A. M. Fuchs, M. V. Balabas, G. Vasilakis, L. D. Mosgaard, H. C. Stærkind, J. H. Müller, T. Heimburg, S.-P. Olesen, and E. S. Polzik, Non-invasive Detection of Animal Nerve Impulses with an Atomic Magnetometer Operating near Quantum Limited Sensitivity, Sci. Rep. 6, 29638 (2016).

[37] J. F. Barry, M. J. Turner, J. M. Schloss, D. R. Glenn, Y. Song, M. D. Lukin, H. Park, and R. L. Walsworth, Optical Magnetic Detection of Single-Neuron Action Potentials Using Quantum Defects in Diamond, Proc. Natl. Acad. Sci. U.S.A. 113, 14133 (2016).

[38] We neglect the hyperfine coupling of the NV electronic spin to nearby nuclear spins. The experiments are performed in the presence of a static bias magnetic field of $B=39.4 \mathrm{mT}$ (see Appendix) aligned along the symmetry axis of the NV center ( $\hat{z}$ axis), where we observe full polarization of the nuclear spin of the nitrogen-14 composing the NV center [39].

[39] F. Poggiali, P. Cappellaro, and N. Fabbri, Measurement of the Excited-State Transverse Hyperfine Coupling in NV Centers via Dynamic Nuclear Polarization, Phys. Rev. B 95, 195308 (2017).

[40] M. W. Doherty, N. B. Manson, P. Delaney, F. Jelezko, J. Wrachtrup, and L. C. Hollenberg, The Nitrogen-Vacancy Colour Centre in Diamond, Phys. Rep. 528, 1 (2013).

[41] M. Tsang, H. M. Wiseman, and C. M. Caves, Fundamental Quantum Limit to Waveform Estimation, Phys. Rev. Lett. 106, 090401 (2011).

[42] Here, we limit the notion of "optimal" control to optimality over this restricted choice of control strategies. We are supported in our choice of strategy by the great success that dynamical decoupling has obtained in quantum sensing. While allowing general control fields could lead to a true optimal solution, it would also complicate the search space and its computational cost. While we do not demonstrate the realization of the ultimate limit of sensitivity, that is, the quantum Crámer-Rao bound [43], our optimal control method remarkably enhances sensitivity in a number of relevant and demanding experimental scenarios.

[43] A. Holevo, Probabilistic and Statistical Aspects of Quantum Theory (North-Holland, Amsterdam, 1982).

[44] A. Ajoy, G. A. Álvarez, and D. Suter, Optimal Pulse Spacing for Dynamical Decoupling in the Presence of a Purely Dephasing Spin Bath, Phys. Rev. A 83, 032303 (2011).

[45] M. J. Biercuk, A.C. Doherty, and H. Uys, Dynamical Decoupling Sequence Construction as a Filter-Design Problem, J. Phys. B 44, 154002 (2011). 
[46] F. Reinhard, F. Shi, N. Zhao, F. Rempp, B. Naydenov, J. Meijer, L. T. Hall, L. Hollenberg, J. Du, R.-B. Liu, and J. Wrachtrup, Tuning a Spin Bath through the QuantumClassical Transition, Phys. Rev. Lett. 108, 200402 (2012).

[47] M. S. Byrd and D. A. Lidar, Comprehensive Encoding and Decoupling Solution to Problems of Decoherence and Design in Solid-State Quantum Computing, Phys. Rev. Lett. 89, 047901 (2002).

[48] J. Bylander, S. Gustavsson, F. Yan, F. Yoshihara, K. Harrabi, G. Fitch, D. G. Cory, and W. D. Oliver, Noise Spectroscopy through Dynamical Decoupling with a Superconducting Flux Qubit, Nat. Phys. 7, 565 (2011).

[49] K. Kakuyanagi, T. Meno, S. Saito, H. Nakano, K. Semba, H. Takayanagi, F. Deppe, and A. Shnirman, Dephasing of a Superconducting Flux Qubit, Phys. Rev. Lett. 98, 047004 (2007).

[50] L. Cywiński, R. M. Lutchyn, C. P. Nave, and S. DasSarma, How to Enhance Dephasing Time in Superconducting Qubits, Phys. Rev. B 77, 174509 (2008).

[51] D. Kielpinski, V. Meyer, M. A. Rowe, C. A. Sackett, W. M. Itano, C. Monroe, and D. J. Wineland, A Decoherence-Free Quantum Memory Using Trapped Ions, Science 291, 1013 (2001).

[52] M. J. Biercuk, H. Uys, A. P. VanDevender, N. Shiga, W. M. Itano, and J. J. Bollinger, Experimental Uhrig Dynamical Decoupling Using Trapped Ions, Phys. Rev. A 79, 062324 (2009).

[53] N. Bar-Gill, L. Pham, C. Belthangady, D. Le Sage, P. Cappellaro, J. Maze, M. Lukin, A. Yacoby, and R. Walsworth, Suppression of Spin-Bath Dynamics for Improved Coherence of Multi-Spin-Qubit Systems, Nat. Commun. 3, 858 (2012).

[54] Y. Romach, C. Müller, T. Unden, L. J. Rogers, T. Isoda, K. M. Itoh, M. Markham, A. Stacey, J. Meijer, S. Pezzagna, B. Naydenov, L. P. McGuinness, N. Bar-Gill, and F. Jelezko, Spectroscopy of Surface-Induced Noise Using Shallow Spins in Diamond, Phys. Rev. Lett. 114, 017601 (2015).

[55] J. R. Petta, A. C. Johnson, J. M. Taylor, E. A. Laird, A. Yacoby, M. D. Lukin, C. M. Marcus, M. P. Hanson, and A. C. Gossard, Coherent Manipulation of Coupled Electron Spins in Semiconductor Quantum Dots, Science 309, 2180 (2005).

[56] G. S. Uhrig, Keeping a Quantum Bit Alive by Optimized $\pi$ Pulse Sequences, Phys. Rev. Lett. 98, 100504 (2007).

[57] G. A. Paz-Silva and L. Viola, General Transfer-Function Approach to Noise Filtering in Open-Loop Quantum Control, Phys. Rev. Lett. 113, 250501 (2014).

[58] S. L. Braunstein and C. M. Caves, Statistical Distance and the Geometry of Quantum States, Phys. Rev. Lett. 72, 3439 (1994).

[59] See Supplemental Material at http://link.aps.org/ supplemental/10.1103/PhysRevX.8.021059 for additional data on multitone ac fields in a broader frequency range, and magnetic impulses with slow temporal profile.

[60] H. Y. Carr and E. M. Purcell, Effects of Diffusion on Free Precession in Nuclear Magnetic Resonance Experiments, Phys. Rev. 94, 630 (1954).

[61] C. P. Slichter, Principles of Magnetic Resonance, 3rd ed. (Springer-Verlag, Berlin, 1996).
[62] L. Viola and S. Lloyd, Dynamical Suppression of Decoherence in Two-State Quantum Systems, Phys. Rev. A 58, 2733 (1998).

[63] J. M. Taylor, P. Cappellaro, L. Childress, L. Jiang, D. Budker, P. R. Hemmer, A. Yacoby, R. Walsworth, and M. D. Lukin, High-Sensitivity Diamond Magnetometer with Nanoscale Resolution, Nat. Phys. 4, 810 (2008).

[64] N. Zhao, J. Honert, B. Schmid, M. Klas, J. Isoya, M. Markham, D. Twitchen, F. Jelezko, R.-B. Liu, H. Fedder, and J. Wrachtrup, Sensing Single Remote Nuclear Spins, Nat. Nanotechnol. 7, 657 (2012).

[65] S. Kolkowitz, A. C. Bleszynski Jayich, Q. P. Unterreithmeier, S. D. Bennett, P. Rabl, J. G. E. Harris, and M. D. Lukin, Coherent Sensing of a Mechanical Resonator with a SingleSpin Qubit, Science 335, 1603 (2012).

[66] T. H. Taminiau, J. J. T. Wagenaar, T. van der Sar, F. Jelezko, V. V. Dobrovitski, and R. Hanson, Detection and Control of Individual Nuclear Spins Using a Weakly Coupled Electron Spin, Phys. Rev. Lett. 109, 137602 (2012).

[67] F. Shi, Q. Zhang, P. Wang, H. Sun, J. Wang, X. Rong, M. Chen, C. Ju, F. Reinhard, H. Chen, J. Wrachtrup, J. Wang, and J. Du, Single-Protein Spin Resonance Spectroscopy under Ambient Conditions, Science 347, 1135 (2015).

[68] For a bare CP sequence, the filter function is $Y_{n, T}=[\sin (\pi \nu T) /(\pi \nu T)(1-\sec (\pi \nu T / n)) \cos (\pi \nu T+\alpha)]^{2}$, which is peaked at $\nu=k / 2 \tau$, with $k$ being an odd integer index, where the higher harmonics have decreasing weight [13].

[69] N. Zhao, J. Wrachtrup, and R.-B. Liu, Dynamical Decoupling Design for Identifying Weakly Coupled Nuclear Spins in a Bath, Phys. Rev. A 90, 032319 (2014).

[70] J. Casanova, Z.-Y. Wang, J. F. Haase, and M. B. Plenio, Robust Dynamical Decoupling Sequences for Individual-Nuclear-Spin Addressing, Phys. Rev. A 92, 042304 (2015).

[71] A. Ajoy, Y.-X. Liu, K. Saha, L. Marseglia, J.-C. Jaskula, U. Bissbort, and P. Cappellaro, Quantum Interpolation for High-Resolution Sensing, Proc. Natl. Acad. Sci. U.S.A. 114, 2149 (2017).

[72] W. Ma, F. Shi, K. Xu, P. Wang, X. Xu, X. Rong, C. Ju, C.-K. Duan, N. Zhao, and J. Du, Resolving Remote Nuclear Spins in a Noisy Bath by Dynamical Decoupling Design, Phys. Rev. A 92, 033418 (2015).

[73] Different multitone ac fields are considered in Ref. [59].

[74] A. M. Schrand, S. A. C. Hens, and O. A. Shenderova, Nanodiamond Particles: Properties and Perspectives for Bioapplications, Crit. Rev. Solid State Mater. Sci. 34, 18 (2009).

[75] A. Laraoui, J. S. Hodges, and C. A. Meriles, NitrogenVacancy-Assisted Magnetometry of Paramagnetic Centers in an Individual Diamond Nanocrystal, Nano Lett. 12, 3477 (2012).

[76] N. Zhao, J.-L. Hu, S.-W. Ho, J. T. K. Wan, and R. B. Liu, Atomic-Scale Magnetometry of Distant Nuclear Spin Clusters via Nitrogen-Vacancy Spin in Diamond., Nat. Nanotechnol. 6, 242 (2011).

[77] F. Shi, X. Kong, P. Wang, F. Kong, N. Zhao, R.-B. Liu, and J. Du, Sensing and Atomic-Scale Structure Analysis of Single Nuclear-Spin Clusters in Diamond, Nat. Phys. 10, 21 (2014). 
[78] T. van der Sar, F. Casola, R. Walsworth, and A. Yacoby, Nanometre-Scale Probing of Spin Waves Using Single Electron Spins, Nat. Commun. 6, 7886 (2015).

[79] T. Yuge, S. Sasaki, and Y. Hirayama, Measurement of the Noise Spectrum Using a Multiple-Pulse Sequence, Phys. Rev. Lett. 107, 170504 (2011).

[80] G. A. Álvarez and D. Suter, Measuring the Spectrum of Colored Noise by Dynamical Decoupling, Phys. Rev. Lett. 107, 230501 (2011).

[81] K. C. Young and K. B. Whaley, Qubits as Spectrometers of Dephasing Noise, Phys. Rev. A 86, 012314 (2012).

[82] L. Faoro and L. Viola, Dynamical Suppression of $1 / f$ Noise Processes in Qubit Systems, Phys. Rev. Lett. 92, 117905 (2004).
[83] I. Almog, Y. Sagi, G. Gordon, G. Bensky, G. Kurizki, and N. Davidson, Direct Measurement of the SystemEnvironment Coupling as a Tool for Understanding Decoherence and Dynamical Decoupling, J. Phys. B 44, 154006 (2011).

[84] M. Loretz, J. M. Boss, T. Rosskopf, H. J. Mamin, D. Rugar, and C. L. Degen, Spurious Harmonic Response of Multipulse Quantum Sensing Sequences, Phys. Rev. X 5, 021009 (2015).

[85] L. Childress, M. V. Gurudev Dutt, J. M. Taylor, A. S. Zibrov, F. Jelezko, J. Wrachtrup, P. R. Hemmer, and M.D. Lukin, Coherent Dynamics of Coupled Electron and Nuclear Spin Qubits in Diamond, Science 314, 281 (2006). 